\title{
Predicting neuropathic ulceration: analysis of static temperature distributions in thermal images
}

\author{
Naima Kaabouch \\ University of North Dakota \\ Electrical Engineering Department \\ Grand Forks, North Dakota 58202
}

\author{
Wen-Chen $\mathrm{Hu}$ \\ University of North Dakota \\ Computer Science Department \\ Grand Forks, North Dakota 58202
}

\section{Yi Chen}

University of North Dakota

Electrical Engineering Department

Grand Forks, North Dakota 58202

\section{Julie W. Anderson}

University of North Dakota

College of Nursing

Grand Forks, North Dakota 58202

\section{Forrest Ames}

University of North Dakota

Mechanical Engineering Department

Grand Forks, North Dakota 58202

\section{Rolf Paulson}

Altru Hospital

Wound Clinic

1200 South Columbia Road

Grand Forks, North Dakota 58201

\begin{abstract}
Foot ulcers affect millions of Americans annually. Conventional methods used to assess skin integrity, including inspection and palpation, may be valuable approaches, but they usually do not detect changes in skin integrity until an ulcer has already developed. We analyze the feasibility of thermal imaging as a technique to assess the integrity of the skin and its many layers. Thermal images are analyzed using an asymmetry analysis, combined with a genetic algorithm, to examine the infrared images for early detection of foot ulcers. Preliminary results show that the proposed technique can reliably and efficiently detect inflammation and hence effectively predict potential ulceration. (C) 2010 Society of Photo-Optical Instrumentation Engineers. [DOI: 10.1117/1.3524233]
\end{abstract}

Keywords: foot ulcers; thermal imaging; asymmetry analysis; automatic thresholding. Paper 10017SSPR received Jan. 14, 2010; revised manuscript received Sep. 1, 2010; accepted for publication Oct. 11, 2010; published online Dec. 23, 2010.

\section{Introduction}

One of the most common mechanisms in the development of foot ulcerations involves the cumulative effect of unrecognized repetitive trauma over the course of several days at pressure points on the sole of the foot. ${ }^{1-3}$ Areas that are likely to ulcerate are those associated with increased local skin temperatures due to inflammation and enzymatic autolysis of tissue. ${ }^{4-6}$ Such inflammation is characterized by five signs: redness, heat, swelling, pain, and loss of function. Some signs are difficult for clinicians to assess objectively; however, temperature measurements can provide quantitative data shown to be predictive of impending ulceration. $^{4,7-10}$

Conventional noninvasive methods used to assess foot skin integrity, including inspection and palpation, may be valuable diagnostic tools, but they usually do not detect changes in skin integrity until skin breakdown has already occurred. Additionally, manual infrared thermometers used in clinics can only provide a mean value over a large area of the foot. In this re-

Address all correspondence to: Naima Kaabouch, University of North Dakota, Electrical Engineering Department, Grand Forks, North Dakota 58202; Tel: 701 777-4460; E-mail: naimakaabouch@mail.und.edu search study, thermal imaging is used to monitor the temperature distribution on the skin. However, there is no standard distribution for skin-surface temperature of a healthy foot because that skin temperature can be affected by many factors, such as ambient and internal thermal conditions, age, sex, weight, etc. One way to eliminate this variability is to compare the thermal skin distributions of two feet for the same subject. ${ }^{11,12}$ This comparison, called asymmetry analysis, is then combined with a segmentation technique that is based on a genetic algorithm to achieve higher efficiency in the detection of inflammation and, hence, better prediction of ulcers before they develop. This proposed methodology involves three steps: segmentation, geometric transformation, and asymmetry analysis.

\section{Methodology}

In this research, a thermal camera, FLIR A320 $\left(0-350^{\circ} \mathrm{C}\right.$, $\pm 2 \%$ ), was used to record foot skin temperature distributions after patients removed their socks and shoes. The resulting thermal images were then analyzed, as illustrated in Fig. 1. This approach, implemented using MATLAB as a platform, involves three steps:

$1083-3668 / 2010 / 15(6) / 061715 / 6 / \$ 25.00$ @ 2010 SPIE 
Kaabouch et al.: Predicting neuropathic ulceration: analysis of static temperature distributions. . .

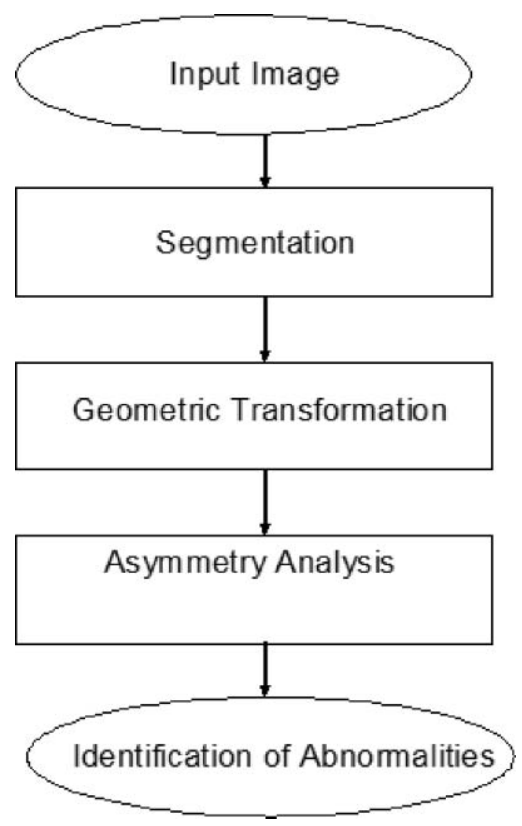

Fig. 1 Flowchart of the proposed methodology.

1. segmentation to extract the feet from the background

2. geometric transformation to adjust the left and right foot, so both feet are in the same position in the image

3. asymmetry analysis to subtract the intensity level of each pixel in the left foot from the intensity level of the symmetric pixel of the right foot and detect potential ulcerous areas

\subsection{Automatic Thresholding}

In this proposed methodology, automatic thresholding of the thermal images is an important step to minimize noise and decrease detection of false inflammation. This step presents certain challenges, however. First, some patients have feet that present areas with low temperatures, which are also close to the temperatures of the image background levels. Second, the heat transfer from the legs and other body parts increases the temperature between the feet, resulting in high nonuniform background levels. Figures 2(a)-2(c) show three examples of thermal images that present nonuniform background levels due to the transfer of heat from the legs and other body parts to the air.
To extract the feet from the background image and remove as much noise as possible, a genetic algorithm is used. Genetic algorithms are optimization algorithms based on biological mechanics of natural selection through certain operations, such as chromosome, population size, cross rate, mutation rate, and maximum generation. ${ }^{12,13}$

Fitness is then evaluated by applying the following equation:

$$
\operatorname{Fitness}(x)=N_{\mathrm{f}} N_{\mathrm{b}}\left(M_{\mathrm{f}}-M_{\mathrm{b}}\right)^{2},
$$

where $N_{\mathrm{f}}$ and $N_{\mathrm{b}}$ are the numbers of foreground and background pixels, respectively.

$M_{\mathrm{f}}$, the mean intensity of foreground pixels, is given by

$$
M_{\mathrm{f}}=\frac{I_{\mathrm{f}}}{N_{\mathrm{f}}},
$$

where $I_{\mathrm{f}}$ is the sum of intensities of foreground pixels.

$M_{\mathrm{b}}$ is the mean intensity of background pixels given by

$$
M_{\mathrm{b}}=\frac{I_{\mathrm{b}}}{N_{\mathrm{b}}},
$$

where $I_{\mathrm{b}}$ is the sum of intensities of background pixels.

The best threshold is determined by applying the following equation:

$$
\operatorname{Fitness}\left(x^{*}\right)=\max \{\operatorname{Fitness}(x)\},
$$

where $x$ represents a threshold level.

The main steps of the proposed genetic algorithm are summarized as follows:

1. assign the length of chromosome, population size, cross rate, mutation rate, and maximum generation

2. initialize the population of the first generation, with each individual being a random eight-bit binary string, representing a specific intensity level

3. evaluate the fitness of the whole population

4. generate the next population by performing selection, crossover, and mutation operations

5. return to step 3 if the desired number of generations is not reached

6. reduce the cross rate and the mutation rate after half of the desired generation number is reached

7. segment the image, using the optimal threshold level when the desired number of generation is reached

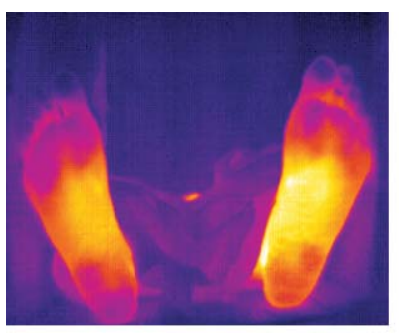

(a)

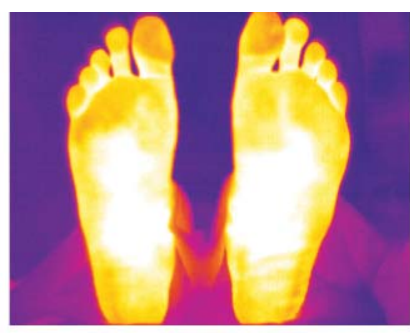

(b)

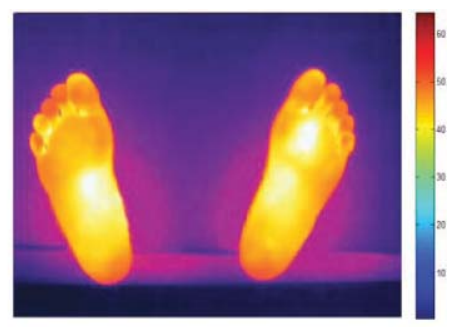

(c)

Fig. 2 Example of thermal images corresponding to (a) healthy feet with cold temperatures areas, (b) healthy feet with nonuniform background due to the heat transfer around the feet, and (c) foot with an abnormal area. 


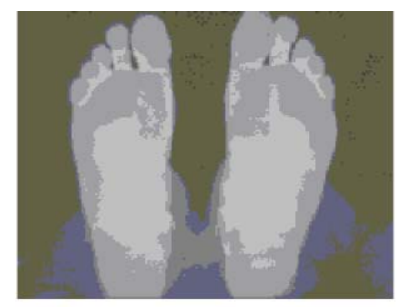

(a)

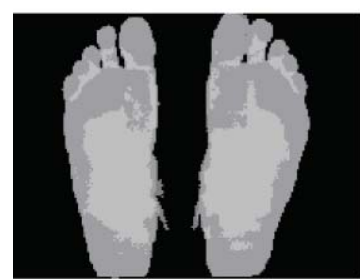

(b)

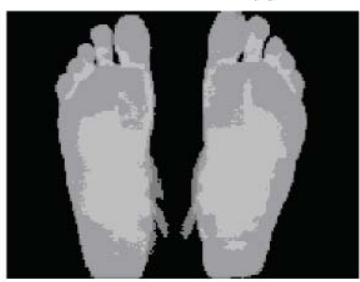

(c)

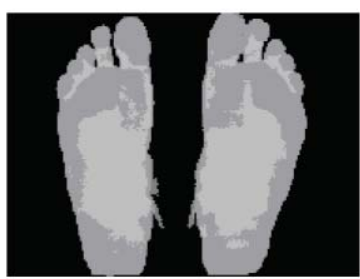

(d)

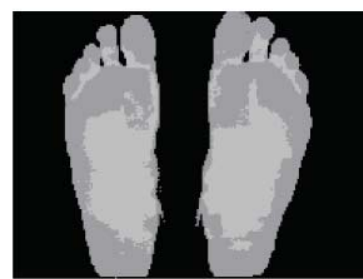

(e)

Fig. 3 Some results of the thresholding techniques: (a) Gray-scale image corresponding to the thermal image of Fig. 2(b), (b) output image after applying moment preserving method, (c) output image after applying maximum entropy method, (d) output image after applying Otsu's method, and (e) output image after applying the genetic algorithm.

\subsection{Geometry Transformation}

The ultimate goal of this research paper is to compare the thermal distributions of a healthy foot to those of an abnormal foot with abnormal areas. For the most accurate comparison, the feet must be arranged symmetrically in relation to each other. However, in practice, feet in an image are rarely in symmetric positions. Therefore, a geometric transformation is needed to adjust their positions. Assuming that the feet in the image are the same size, a simple translation and rotation around two feature points can achieve this goal. The centroid and the farthest point from the centroid to the heel edge serve as reference points for the adjustment of each foot. On the basis of these two feature points, the relative position and angle differences between the two feet are then calculated. Then, a rotation and a translation are performed to set each foot in the middle of the image with the characteristic line in a vertical direction. The geometric transformation procedure is described as follows:

1. separate the original image into two images that correspond to the left foot and right foot

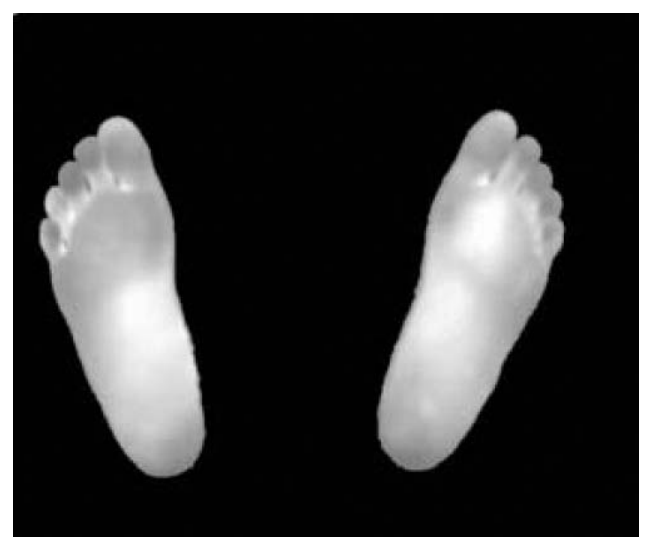

Fig. 4 Output image after applying the genetic algorithm on Fig. 1(c).
2. identify the centroid and farthest heel points of each foot

3. calculate the angle of the characteristic line with respect to the vertical direction

4. perform the translation and the rotation to arrange the feet in the middle of the image: First, each foot is shifted by moving the position of all pixels inside the foot to the center of the image and, then, each foot is rotated to place the characteristic line in a vertical direction.

\subsection{Asymmetry Analysis}

In this step, the potential ulcerous areas are identified by analyzing the asymmetry between the adjusted foot images. Once the feet are adjusted in symmetric positions using the geometric transformation algorithm, the left-foot pixel intensities are subtracted from the corresponding right-foot pixel intensities. If the difference exceeds a specific threshold, then the intensity is shown as an abnormality in image difference.
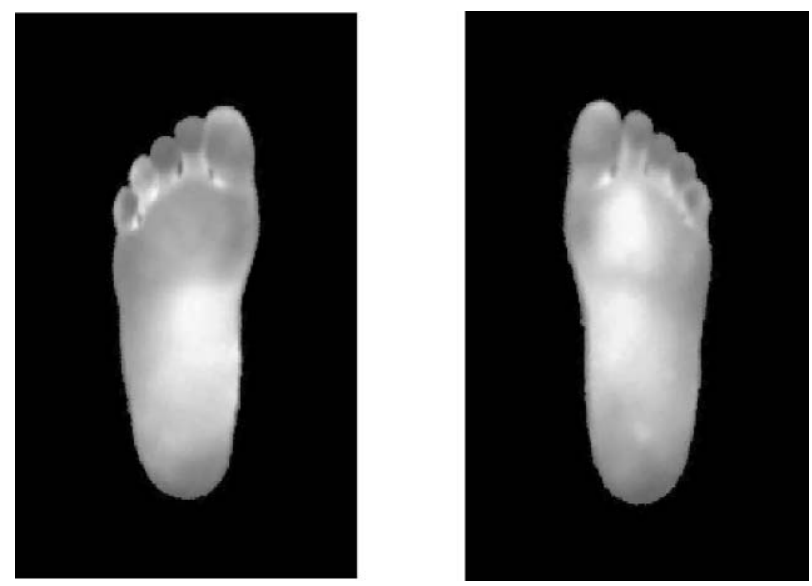

Fig. 5 Output images after applying the geometry transformation technique on Fig. 4. 
Kaabouch et al.: Predicting neuropathic ulceration: analysis of static temperature distributions...

Table 1 Evaluation of different thresholding techniques.

\begin{tabular}{lcccccc}
\hline & Threshold & ME & NU & RAE & EMM & Combination \\
\hline Moment & 135 & 0.019648 & 0.05575 & 0.042063 & 0.047407 & 0.0412 \\
Entropy & 127 & 0.0054818 & 0.039365 & 0.012102 & 0.020619 & 0.0194 \\
Iterative & 133 & 0.010271 & 0.041593 & 0.028223 & 0.031277 & 0.0278 \\
Otsu's method & 127 & 0.0054818 & 0.039365 & 0.012102 & 0.020619 & 0.0194 \\
Genetic & 131 & 0.0028776 & 0.034485 & 0.000437 & 0.013672 & 0.0129 \\
\hline
\end{tabular}

\section{Results}

To assess the effectiveness of this developed technique, we accessed over 100 thermal images, corresponding to patients' feet with and without inflammation. Examples of these thermal images are presented in Figs. 2(a)-2(c). Figures 2(a) and 2(b) show examples of thermal images of healthy feet, whereas Fig. 2(c) shows an example of a thermal image in which the right foot contains a high-temperature area, indicating the presence of inflammation. As seen in these images, the heat transfer from the legs in the thermal images generates a nonuniform background.

An example of results when applying the genetic algorithm is shown in Fig. 3(a). These results are compared to those of other existing techniques that were also implemented, including Otsu's technique, ${ }^{14}$ Ridler and Calvard's technique, ${ }^{15}$ Kapur et al. ${ }^{16}$ and Tsai's technique. ${ }^{17}$ The results of applying these techniques are shown in Figs. 3(b)-3(e). A comparison of all these results is done through use of four criteria as well as their combination. These four criteria are misclassification error (ME), edge mismatch (EMM), relative foreground area error (RAE), and region nonuniformity (NU). The descriptions of the criteria can be found in Refs. 18-21.

Examples of results of this assessment are given in Table 1 , offering the quantification results of the above criteria for the five implemented techniques as applied to the image of Fig. 2(a). From Table 1 one can quantitatively see that the genetic algorithm has a relatively smaller overall combination value than do the other techniques, even though it does not give the best value for every criterion. Similar results are found for the set of thresholded images, which indicate that the genetic algorithm provides better thresholding results than do the other implemented techniques.

Although the genetic algorithm does give better results, it does not completely remove the noise between the feet. One solution to increase this efficiency is separating the feet from the other parts of the body by using a lightweight metallically painted shield. An example of these results is shown in Fig. 4. As

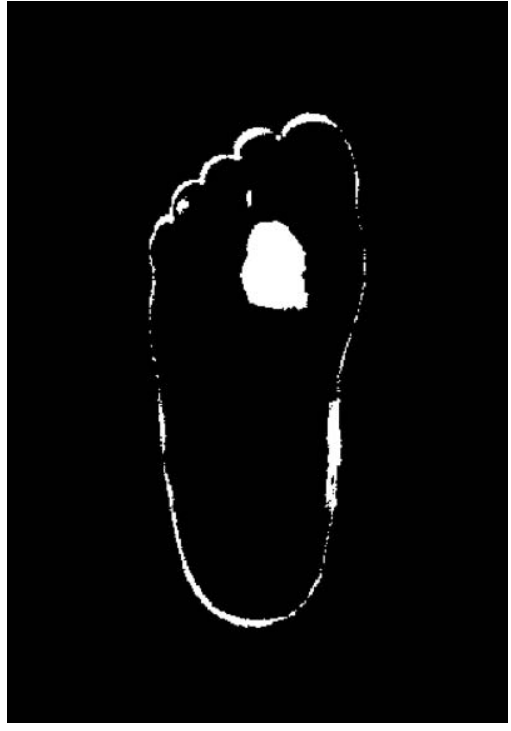

Fig. 6 Output image resulting from the asymmetry analysis of Fig. 5.

observed, the quality of the thresholded image greatly improves and the noise around the feet is completely removed. Figure 5 represents output images with adjusted feet after applying the geometric transformation to the thresholded image of Fig. 4. Figure 6 shows the gray-scale image that represents the result of the asymmetry analysis. In this image, the white spot represents the location of the abnormal area.

Figure 7 shows the results of the asymmetry analysis (output images after overlapping and subtracting) for different inflammation diameters, measured using a ruler and ranging from 1 to $0.2381 \mathrm{~cm}$. As can be seen, inflammation with a diameter as small as $2 \mathrm{~mm}$ can be detected. Furthermore, Figs. 8 and 9 illustrate the accuracy of the technique by showing the linear relationships between the inflammation areas and their corresponding spot areas in those images resulting from the

Table 2 Results of the assessment.

\begin{tabular}{lcccc}
\hline $\begin{array}{l}\text { Number of } \\
\text { images analyze }\end{array}$ & $\begin{array}{c}\text { Number of images } \\
\text { without hot spots }\end{array}$ & $\begin{array}{c}\text { Number of images } \\
\text { with hot spots }\end{array}$ & $\begin{array}{c}\text { Number of images } \\
\text { with false spots }\end{array}$ & $\begin{array}{c}\text { Number of images } \\
\text { with missing spots }\end{array}$ \\
\hline 100 & 55 & 45 & 10 & 0 \\
$100 \%$ & $55 \%$ & $45 \%$ & $10 \%$ & $0 \%$ \\
\hline
\end{tabular}




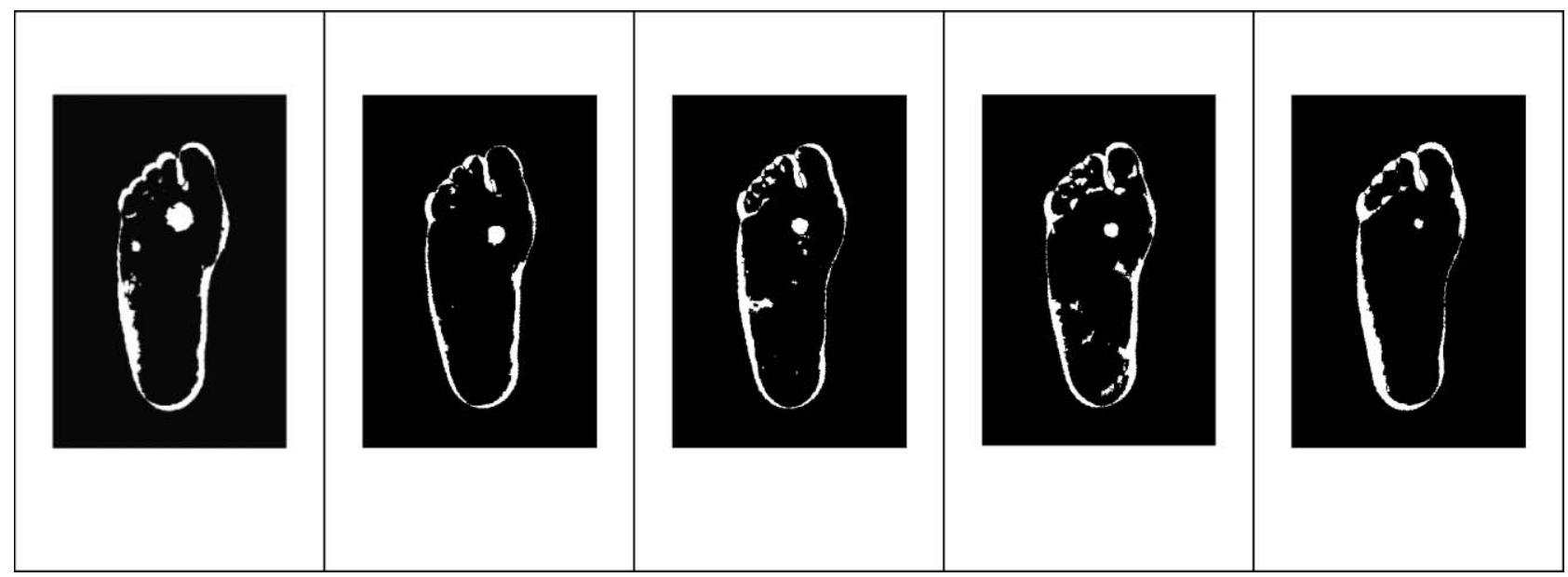

Fig. 7 Results of the asymmetry analysis corresponding to different inflammation diameters.

asymmetry analyses. This relationship will allow clinicians to accurately estimate the area of the inflammation and take corrective actions before an ulcer develops.

The assessment of the efficiency of the proposed system is based on the number of images that show hot spots (feet with or without abnormal areas), the number of images with missing spots (feet with abnormal areas that were not detected), and the number of images with false spots (feet that do not contain abnormal areas). Table 2 offers a summary of such assessment based on these parameters. As one can see, 45 images contained hot spots after analysis with the proposed system; however, 10 of these images were false spots. These false spots were identified as a result of the differences between the shapes and sizes of the left and right feet in the images. Such differences are mainly due to the fact that the patients moved their feet in all directions, and thus, the projections of their feet were not the same in some images.

\section{Conclusion}

We developed a technique to use for investigating the thermal distributions of feet in order to detect inflammation and hence

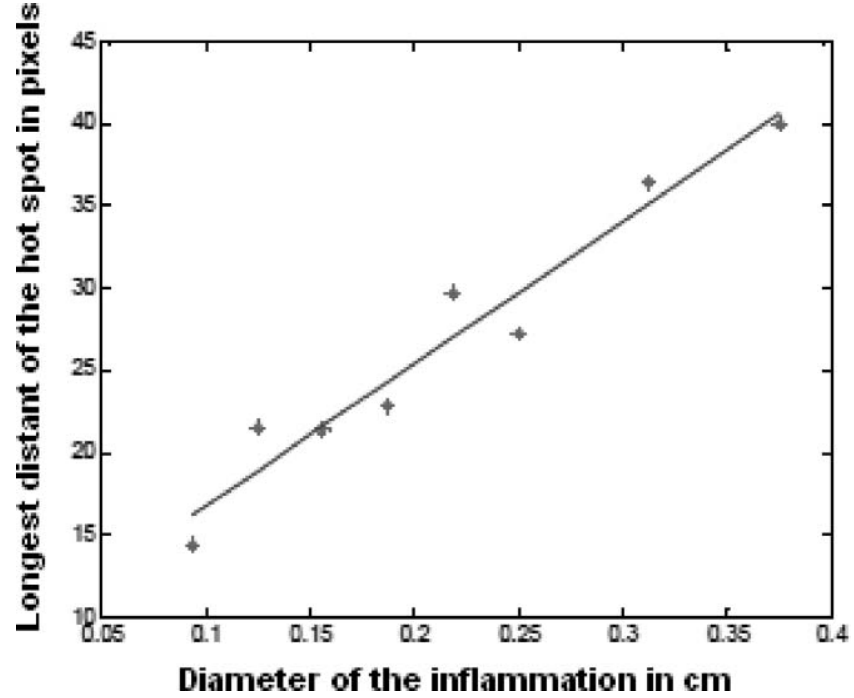

Fig. 9 Width of the inflammation versus width of the spot in the image.

predict foot ulcers. The experimental results indeed show that this technique can be used to identify inflammation and therefore

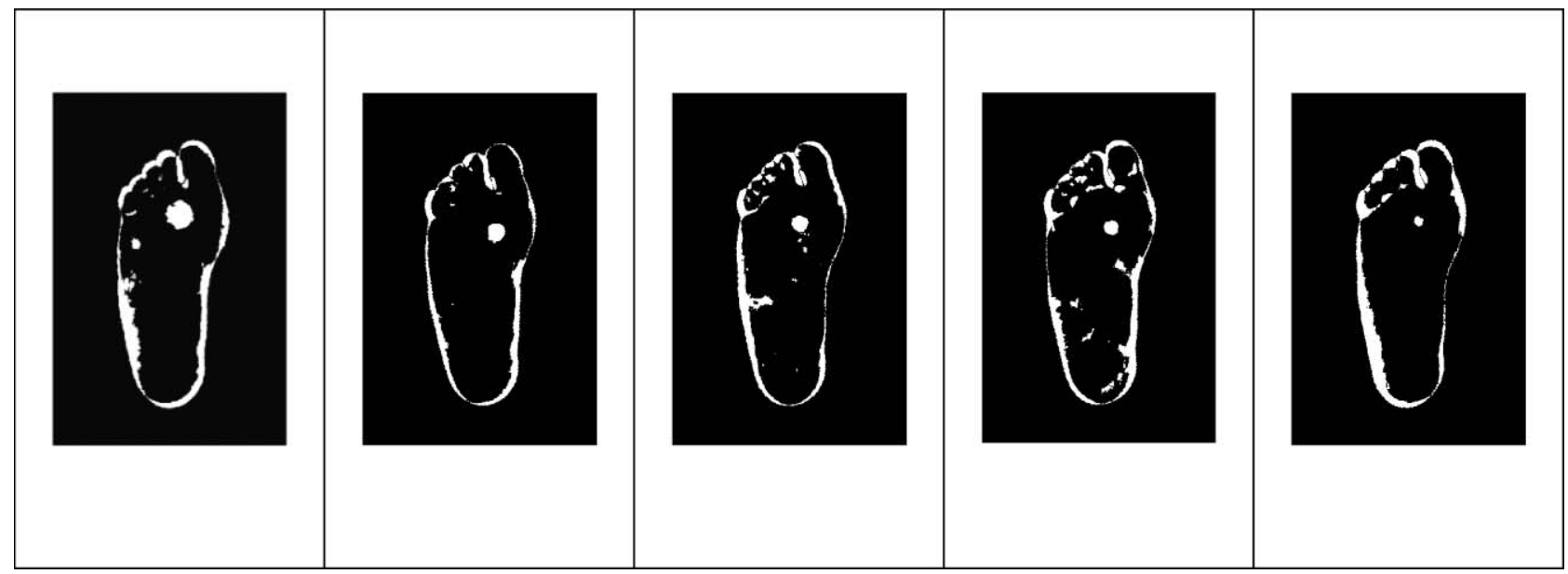

Fig. 8 Area of the inflammation versus area of the spot. 
predict potential ulceration. However, the developed technique assumes that the two feet are on the same plane in an image and have the same shape and size. In the future, our research objectives will include $(i)$ developing a technique that scans and compares symmetric pixels, no matter the shape, size, and position of the feet and (ii) studying the decay rates of temperature distributions over time for successive images that correspond to the same feet.

\section{Acknowledgment}

This work was supported by the ND EPSCoR project through National Science Foundation Grant No. UND0012168.

\section{References}

1. L. A. Lavery, D. G. Armstrong, S. Vela, and J. G. Fleishli, "Practical criteria to screen patients at risk for diabetic foot ulceration," Arch. Int. Med. 158, 157-162 (1998).

2. G. E. Reiber, L. Vileikyte, E. J. Boyko, M. del Aguila, D. G. Smith, L. A. Lavery, and A. J. Boulton, "Causal pathways for incident lower extremity ulcers in patients with diabetes from two settings," Diabetes Care 22, 157-162 (1999).

3. R. G. Frykberg, L. A. Lavery, H. Pham, C. Harvey, L. Harkless, and A. Veves, "Role of neuropathy and high foot pressures in diabetic foot ulceration," Diabetes Care 21, 1714-1719 (1998).

4. D. G. Armstrong, L. A. Lavery, P. J. Liswood, W. F. Todd, and J. Tredwell, "Infrared dermal thermometry of the high-risk diabetic foot," Phys Ther 77, 169-177 (1997).

5. P. W. Brand, "The insensitive foot (including leprosy)," in Disorders of the Foot and Ankle, M. Jahss Ed., Saunders Philadelphia, pp. 2173 2175 (1991).

6. R. P. Clark, M. R. Goff, J. Hughes, and L. Klenerman, "Thermography and pedobarography in the assessment of tissue damage in neuropathic and atherosclerotic feet," Thermology 3, 15-20 (1988).

7. G. E. Reiber, L. Vileikyte, E. J. Boyko, M. del Aguila, D. G. Smith, L. A. Lavery, and A. J. Boulton, "Home monitoring of foot skin temperatures to prevent ulceration," Diabetes Care 27, 2642-2647 (2004).

8. V. Schubert and B. Fagrell, "Local skin pressure and its effect on skin microcirculation as evaluated by laser Doppler fluxmetry," Clin. Physiol. 9, 535-545 (1989).

9. V. Schubert and B. Fagrell, "Evaluation of the dynamic cutaneous post ischaemichyperaemia and thermal response in elderly subjects and in an area at risk for pressure sores," Clin. Physiol. 11, 169-182 (1991).

10. D. G. Armstrong and L. A. Lavery, "Predicting neuropathic ulceration with infrared dermal thermometry," J. Am. Podiatr. Med. Assoc. 87, 336-337 (1997).

11. N. Kaabouch, Y. Chen, W. C. Hu, J. Anderson, F. Ames, and R. Paulson, "Early detection of foot ulcers through asymmetry analysis," Proc. SPIE, $\mathbf{7 2 4 3} 72621 \mathrm{~L}$ (2009).

12. N. Kaabouch, Y. Chen, J. Anderson, F. Ames, and R. Paulson, "Asymmetry analysis based on Genetic Algorithms for the Prediction of Foot Ulcers," Proc. SPIE, 7243 (2009).

13. D. E. Goldber, "Genetic algorithms in search, optimization and machine learning," Addison-Wesley, 1989.

14. N. Otsu, "A threshold selection method from gray level histograms," IEEE Trans. Syst. Man Cybern. SMC-9, 62-66 (1979).

15. T. W. Ridler and S. Calvard, "Picture thresholding using an iterative selection method," IEEE Trans. Syst. Man Cybern. SMC-8, 630-632 (1978).

16. J. N. Kapur, P. K. Sahoo, and A. K. C. Wong, "A new method for graylevel picture thresholding using the entropy of the histogram," Graph. Models Image Process. 29, 273-285 (1985).

17. W. H. Tsai, "Moment-preserving thresholding: a new approach," Graph. Models Image Process. 19, 377-393 (1985).

18. M. Sezgin and B. Sankur, "Survey over image thresholding techniques and quantitative performance evaluatio," J. Electronique Imaging 13(1), 146-165 (2004).

19. Y. J. Zhang, "A survey on evaluation methods for image segmentation," Pattern Recogn. 29, 1335-1346 (1996).

20. W. A. Yasnoff, J. K. Mui, and J. W. Bacus, "Error measures for scene segmentation," Pattern Recogn. 9, 217-231 (1977).

21. M. D. Levine and A. M. Nazif, "Dynamic measurement of computer generated image segmentations," IEEE Trans. Patt. Anal. Mach. Intel. 7(2), 155-164 (1985). 